Acta Cryst. (2002). A58 (Supplement), C161

\section{FEATURES OF STRUCTURE AND COMPOSITION OF THE GLASSES OF KARA DEPRESSION}

A. Lysiuk

Instytute of Geology Komi Scientific Center Pervomaiscaja 54 SYKTYVKAR 167892 RUSSIA

Impact glasses distributed in zuvites of Kara crater, for a long time attracts attention of the experts, because they are the important indicator of processes of shock metamorphism and its research opens new prospects in study of a history of development of the Earth. On morphological features, chemical structure and physical properties impact glasses are subdivided into two groups. The first group makes most widespread light glasses. The presence of the brightly expressed secondary changes, frequently up to a condition similar to clay is characteristic for them. In weathering condition they have white coloring. Less widespread dark glasses with variations of color from cherry up to black concern to the second group of impact glasses. Among them the versions practically of not changed glasses of black coloring with inclusions of quartz, fieldspat, diaplectic glasses are allocated. The secondary changes are expressed poorly. Under the contents of water in the impactites allocate two types of glasses. In black glasses its contents do not exceed $2 \%$, and in light achieves $8-10 \%$. In dark glasses - adsorptions and structural - free water contains from 3 up to $5 \%$, and crystallized from 0,2 up to $2 \%$. In light glasses the contents of structural - free water achieves 7-9\%. As the information on a condition and amount of molecular water in impact glasses can open a nature of the impactites, the given problem is extremely important and requires the further detailed research.

\section{Keywords: GLASSES IMPACT CRATER}

Acta Cryst. (2002). A58 (Supplement), C161

\section{A CLEVER METHOD OF QUANTITATIVE ANALYSIS OF} ALUMINUM-RICH MULLITE

X. $\mathrm{Yao}^{1} \mathrm{Hao}^{2}$

${ }^{1}$ Nankai University Central Laboratory Central Laboratory of Nankai University TIANJIN 300071 CHINA ${ }^{2}$ Tianjin Electronic Materials Research Institute, Tianjin, 300192, China

Mullite is an important ceramic material. Its typical chemical composition is $\left[3\left(\mathrm{Al}_{2} \mathrm{O}_{3}\right) 2\left(\mathrm{SiO}_{2}\right)\right]$. In fact, frequently the actual chemical composition of mullite samples deviates from this ideal value owing to the different preparation technology, but its structure framework is still invariable. It is often demanded to determine rapidly and accurately the content of mullite in a mixture system, which is composed of polycrystalline mullite and some amorphous products in preparation. The difficulty lies in that the intensities of diffraction peaks of the mullite phase are variable along with the different ratio of $\mathrm{Al}$ and $\mathrm{Si}$ atoms of the mullite phase.

Using structure parameters of different aluminum-rich mullites, we caculated their $\mathrm{K}(\mathrm{hkl})$ values. $\mathrm{K}(\mathrm{hkl})=\mathrm{M}(\mathrm{hkl})[$ Square $|\mathrm{F}(\mathrm{hkl})|] \mathrm{Lp}(\mathrm{hkl})$. Here $\mathrm{F}(\mathrm{hkl})$ is the structure factor of (hkl) reflection, $\mathrm{M}(\mathrm{hkl})$ is the multiplicity factor and $L p(h k l)$ is the $L p$ factor. The $K(h k l)$ value of every hkl reflection is variable along with the different ratio of $\mathrm{Al} / \mathrm{Si}$. We selected and analyzed those reflections, the $\mathrm{K}(\mathrm{hkl})$ value of which changes linearly along with the ratio of $\mathrm{Al} / \mathrm{Si}$. We found that the combination of the integrate intensity of three reflections $\mathrm{I}(100)+0.56[\mathrm{I}(120)+\mathrm{I}(210)]$ is not variable. Provided the integrate intensity of these three reflections of samples is determined, the quantitative analysis of mullite can be completed rapidly and accurately.

\section{Keywords: MULLITE POWDER DIFFRACTION QUANTITATIVE} ANALYSIS

\section{Acta Cryst. (2002). A58 (Supplement), C161 \\ ASYMMETRY EFFECTS IN DIFFRACTION BY DISORDERED CHARGE-DENSITY WAVE SYSTEMS}

S. Ravy ${ }^{1}$ J.-P. Pouget ${ }^{1}$ S. Rouziere ${ }^{2}$ S. Brazovskii ${ }^{3}$

${ }^{1}$ Cnrs Umr8502 Laboratoire De Physique Des Solides, Bat. 510 Universite Paris-Sud ORSAY 91405 FRANCE ${ }^{2}$ LURE, Universite Paris-sud, 91405 Orsay, France ${ }^{3}$ Laboratoire de Physique Theorique et Modeles Statistiques, Universite Paris-sud, 91405 Orsay, France

The aim of this paper is to present odd diffraction effects related to impurities in charge-density wave systems. Starting from the study of organic conductors from the TTF-TCNQ family, where white diffuse lines were first observed, we will show that the asymmetry can provide essential information on pinned charge-density waves in low dimensional systems. It will be shown that in the limit of small concentration of impurities, this intensity asymmetry can be interpreted as an "holographic diffraction" effect. Then, another type of asymmetry will be discussed, related to the presence of Friedel oscillations around charged impurities in $\mathrm{K}_{0.3} \mathrm{MoO}_{3}$ blue bronzes.

\section{Keywords: CHARGE DENSITY WAVE DIFFUSE SCATTERING DISORDER}

\section{Acta Cryst. (2002). A58 (Supplement), C161 \\ THE LIQUID-SOLID TRANSITION IN A MICELLAR SOLUTION OF A DIBLOCK COPOLYMER IN WATER \\ V. Castelletto ${ }^{1}$ C. Caillet ${ }^{1}$ I.W. Hamley ${ }^{1}$ Z. Yang ${ }^{2}$ A. Kelarakis ${ }^{3}$ \\ ${ }^{1}$ University of Leeds School of Chemistry LEEDS WEST YORKSHIRE LS8 9JT UK \\ ${ }^{2}$ Department of Chemistry, University of Manchester, Manchester M13 9PL, UK. ${ }^{3}$ National and Kapodistrian University of Athens, Department of Chemistry. Panepistimiopolis 15771 Athens, Greece.}

The structure of a diblock copolymer solution in the vicinity of the transition between micellar liquid and solid phases was investigated using small-angle X-ray scattering (SAXS). An amphiphilic poly(oxyethylene)poly(oxybutylene) diblock was studied in water. Static and dynamic light scattering techniques provided an independent measure of micelle dimensions and aggregation numbers. Phase transitions were located by dynamic shear rheometry and mobility measurements. A micellar liquid phase was identified at low concentration and a cubic micellar phase at higher concentration, the transition between the two occurring at higher temperature as the concentration increased. Intermediate between these two phases, a so-called 'soft gel' phase was observed, this being a viscoelastic fluid but with a much lower dynamic modulus than the hard gel. We analysed the structure of solutions of our diblock copolymer via detailed model fits to the SAXS data for concentrations spanning the liquid- hard gel transition. The micellar form factor was modeled as a homogeneous micellar core with attached Gaussian chains; and the intermicellar structure factor could be described using the hard sphere model. The effective volume fractions determined from the hard sphere structure factor indicate that the 'soft gel' is simply a coexistence region between regions of hard sphere fluid and solid crystal phases. It is apparent that block copolymer micelles act as model colloidal systems in which it is possible to investigate the influence of attractive and repulsive interactions between spherical particles by varying the copolymer composition.

Keywords: POLYMERS, SAXS, RHEOLOGY 